\title{
Association of onset to balloon and door to balloon time with long term clinical outcome in patients with ST elevation acute myocardial infarction having primary percutaneous coronary intervention: observational study \\ (c) (1) \& $\mathrm{gY}$ OPEN ACCESS
}

Hiroki Shiomi MD/PhD candidate ${ }^{1}$, Yoshihisa Nakagawa director ${ }^{2}$, Takeshi Morimoto professor ${ }^{3}$, Yutaka Furukawa director ${ }^{4}$, Akira Nakano associate professor ${ }^{5}$, Shinichi Shirai cardiologist ${ }^{6}$, Ryoji Taniguchi cardiologist ${ }^{7}$, Kyohei Yamaji cardiologist ${ }^{6}$, Kazuya Nagao cardiologist ${ }^{8}$, Tamaki Suyama cardiologist $^{9}$, Hirokazu Mitsuoka cardiologist ${ }^{10}$, Makoto Araki cardiologist ${ }^{11}$, Hiroyuki Takashima assistant professor ${ }^{12}$, Tetsu Mizoguchi cardiologist ${ }^{13}$, Hiroshi Eisawa director ${ }^{14}$, Seigo Sugiyama associate professor $^{15}$, Takeshi Kimura professor ${ }^{1}$, on behalf of the CREDO-Kyoto AMI investigators

${ }^{1}$ Department of Cardiovascular Medicine, Graduate School of Medicine, Kyoto University, Kyoto, 606-8507, Japan; ${ }^{2}$ Division of Cardiology, Tenri Hospital, 200 Mishimacho, Tenri city, Nara, 632-8552, Japan; ${ }^{3}$ Center for General Internal Medicine and Emergency Care, Kinki University School of Medicine, Sayama-city, Osaka 589-8511, Japan; ${ }^{4}$ Division of Cardiology, Kobe City Medical Center General Hospital, Chuo-ku, Kobe, 650-0046, Japan; ${ }^{5}$ Division of Cardiology, University of Fukui Hospital, Yoshidagun, Fukui, 910-1193, Japan; ${ }^{6}$ Division of Cardiology, Kokura Memorial Hospital, Kitakyushu, Fukuoka, 802-8555, Japan; ${ }^{7}$ Division of Cardiology, Hyogo Prefectural Amagasaki Hospital, Amagasaki, Hyogo, 660-0828, Japan; ${ }^{8}$ Division of Cardiology, Osaka Red Cross Hospital, Tennoji-ku, Osaka, 543-8555, Japan; ${ }^{9}$ Division of Cardiology, Sapporo Higashi Tokushukai Hospital, Higashi-ku, Sapporo, 065-0033, Japan; ${ }^{10}$ Division of Cardiology, Kishiwada City Hospital, Kishiwada, Osaka, 596-8501, Japan; ${ }^{11}$ Division of Cardiology, Shimada Municipal Hospital, Shimada, Shizuoka 427-8502, Japan; ${ }^{12}$ Department of Cardiovascular and Respiratory Medicine, Shiga University of Medical Science Hospital, Ostu, Shiga, 520-2192, Japan; ${ }^{13}$ Division of Cardiology, Mitsubishi Kyoto Hospital, Nishigyo-ku Kyoto, 615-8087, Japan; ${ }^{14}$ Division of Cardiology, Nishi-Kobe Medical Center, Nishi-ku, Kobe, 651-2273, Japan; ${ }^{15}$ Department of Cardiovascular Medicine, Graduate School of Medical Sciences, Kumamoto University, Honjo, Kumamoto, 860-8556, Japan

\begin{abstract}
Objective To evaluate the relation of symptom onset to balloon time and door to balloon time with long term clinical outcome in patients with ST segment elevation myocardial infarction (STEMI) having primary percutaneous coronary intervention.

Design Observation of large cohort of patients with acute myocardial infarction.

Setting 26 tertiary hospitals in Japan.

Participants 3391 patients with STEMI who had primary percutaneous coronary intervention within 24 hours of symptom onset.

Main outcome measures Composite of death and congestive heart failure, compared by onset to balloon time and door to balloon time.
\end{abstract}

Results Compared with an onset to balloon time greater than 3 hours, a time of less than 3 hours was associated with a lower incidence of a composite of death and congestive heart failure $(13.5 \%(123 / 964) v$ $19.2 \%$ (429/2427), $P<0.001$; relative risk reduction 29.7\%). After adjustment for confounders, a short onset to balloon time was independently associated with a lower risk of the composite endpoint (adjusted hazard ratio $0.70,95 \%$ confidence interval 0.56 to 0.88 ; $\mathrm{P}=0.002)$. However, no significant difference was found in the incidence of a composite of death and congestive heart failure between the two groups of patients with short ( $\leq 90$ minutes) and long ( $>90$ minutes) door to balloon time $(16.7 \%(270 / 1671) v 18.4 \%(282 / 1720), P=0.54$; relative risk reduction $9.2 \%)$. After adjustment for confounders, no significant difference was seen in the risk of the composite endpoint between the two groups of patients with short and long door to balloon time (adjusted hazard ratio: $0.98,0.78$ to $1.24: P=0.87$ ). A door to balloon time of less 
than 90 minutes was associated with a lower incidence of a composite of death and congestive heart failure in patients who presented within 2 hours of symptom onset $(11.9 \%(74 / 883) v 18.1 \%$ (147/655), $\mathrm{P}=0.01$; relative risk reduction $34.3 \%$ ) but not in patients who presented later $(19.7 \%(196 / 788) v 18.7 \%(135 / 1065), P=0.44 ;-5.3 \%)$. Short door to balloon time was independently associated with a lower risk of a composite of death and congestive heart failure in patients with early presentation (adjusted hazard ratio $0.58,0.38$ to $0.87 ; \mathrm{P}=0.009$ ) but not in patients with delayed presentation $(1.57,1.12$ to $2.18 ; \mathrm{P}=0.008)$. A significant interaction was seen between door to balloon time and time to presentation (interaction $\mathrm{P}=0.01$ ).

Conclusions Short onset to balloon time was associated with better 3 year clinical outcome in patients with STEMI having primary percutaneous coronary intervention, whereas the benefit of short door to balloon time was limited to patients who presented early. Efforts to minimise onset to balloon time, including reduction of patient related delay, should be recommended to improve clinical outcome in STEMI patients.

\section{Introduction}

Primary percutaneous coronary intervention is considered to play a central role in the treatment of ST segment elevation myocardial infarction (STEMI). ${ }^{12}$ The American College of Cardiology/American Heart Association guideline for STEMI recommends primary percutaneous coronary intervention within 90 minutes of first medical contact, or door to balloon time, as a class 1 recommendation and also recommends a total ischaemic time within 120 minutes. ${ }^{34}$ The European Society of Cardiology guideline for STEMI recommends primary percutaneous coronary intervention within 120 minutes of first medical contact in all cases and within 90 minutes in patients presenting early (less than two hours) with a large infarct and low risk of bleeding. ${ }^{5}$ However, reports from previous studies are inconsistent regarding the effect of onset to balloon and door to balloon times on clinical outcomes in patients with STEMI having primary percutaneous coronary intervention. ${ }^{6-9}$ Several relatively small studies suggested a positive correlation between short onset to balloon time and decreased mortality, ${ }^{89}$ whereas the largest study, which enrolled more than 20000 patients (national registry of myocardial infarction in the United States), found that in-hospital mortality did not increase with increasing delay in onset to balloon time time.${ }^{67}$ Furthermore, short door to balloon time was associated with lower in-hospital mortality regardless of time from symptom onset to presentation in the US study, ${ }^{67}$ whereas several other registries have reported the benefit of short door to balloon time to be limited to patients with early presentation. ${ }^{10-12}$

In an attempt to resolve these conflicts about the onset to balloon time and door to balloon time, we evaluated the relation between the two times and the long term clinical outcome of patients with STEMI in a large observational database of patients with acute myocardial infarction in Japan.

\section{Methods}

\section{Study population}

The Coronary REvascularization Demonstrating Outcome Study in Kyoto (CREDO-Kyoto) acute myocardial infarction registry is a physician initiated, non-company sponsored, multicentre registry that enrolled consecutive patients with acute myocardial infarction having coronary revascularisation within seven days of the onset of symptoms between January 2005 and December 2007 across 26 tertiary hospitals in Japan (supplementary appendix A). The relevant review boards or ethics committees in all 26 participating centres approved the study protocol. Obtaining written informed consent from the patients was unnecessary because of the retrospective nature of the study; however, we excluded those patients who refused participation in the study when contacted at follow-up.

Among 5429 patients enrolled in the CREDO-Kyoto acute myocardial infarction registry, 4444 patients had an admission diagnosis of STEMI and had primary percutaneous coronary intervention, excluding 195 patients who had coronary artery bypass grafting surgery and 790 patients with non-STEMI. The population for this study consisted of the 3391 patients with STEMI who had primary percutaneous coronary intervention within 24 hours of onset and for whom data on onset to balloon time were available (fig $1 \Downarrow$ ).

Independent experienced clinical research coordinators from the study management centre collected demographic, angiographic, and procedural data from hospital charts or hospital databases according to pre-specified definitions. They obtained follow-up data from hospital charts or by contacting patients or referring physicians. The median length of follow-up was 1049 (interquartile range 752-1324) days. Complete one year follow-up information was available for 3240 (95.5\%) of the 3391 study patients.

\section{Definitions and endpoints}

We defined onset to balloon time as the time from onset of symptoms to first balloon inflation during percutaneous coronary intervention. We defined door to balloon time as the time from arrival at the hospital to first balloon inflation during percutaneous coronary intervention, and presentation time denoted the time from symptom onset to arrival at the hospital (fig $2 \Downarrow$ ).

The principal outcome measure for this analysis was a composite of death and congestive heart failure. We defined congestive heart failure as admission to hospital for worsening heart failure requiring intravenous drug treatment. We assessed clinical outcomes both at 30 days and up to three years after the onset of STEMI according to onset to balloon time and door to balloon time.

We divided the patients into two groups according to their onset to balloon time to assess the effect of a short onset to balloon time on clinical outcomes. We selected a cut-off point of three hours for short onset to balloon time in light of previously reported data and the distribution of onset to balloon time in this cohort (fig $3 \Downarrow$, left panel). ${ }^{13} \mathrm{We}$ assessed the effect of short onset to balloon time ( $\leq 3$ hours) on clinical outcomes in the entire cohort. We also assessed clinical outcomes according to four categories of onset to balloon time ( $\leq 3$ hours, 3-6 hours, 6-12 hours, and 12-24 hours). As a subgroup analysis, we compared the effects of short onset to balloon time on the incidences of a composite of death and congestive heart failure among the subgroups with or without risk factors such as heart failure, advanced age, anterior myocardial infarction, renal insufficiency, and multivessel disease.

We also divided the patients into two groups according door to balloon time. We set the cut-off point of 90 minutes for short door to balloon time according to the recommendation in the ACC/AHA guideline for STEMI. ${ }^{3} \mathrm{We}$ assessed the effect of short door to balloon time ( $\leq 90$ minutes) on the principal outcome measure in the entire cohort and in the subgroups of patients with early presentation ( $\leq 2$ hours after symptom onset) and delayed presentation ( $>2$ hours after symptom onset). We selected the cut-off point of two hours for early presentation in light of previously reported data and the distribution of the presentation time in this cohort (fig $3 \Downarrow$, right panel). ${ }^{5}{ }^{1011}$ 


\section{Statistical analysis}

We present continuous variables as the mean and standard deviation or median and interquartile range and categorical variables as numbers and percentages. We compared categorical variables with the $\chi^{2}$ test when appropriate; otherwise, we used Fisher's exact test. We compared continuous variables with Student's $t$ test or a Wilcoxon rank-sum test on the basis of the distribution. We used the Kaplan-Meier method to estimate cumulative incidences of clinical event rates and assessed differences with the log-rank test.

We used a multivariable Cox proportional hazards model stratified by centres to estimate the hazard ratio of short onset to balloon time for a composite of death and congestive heart failure by adjusting for 41 clinically relevant variables (listed in supplementary table A). We dichotomised the continuous variables such as age or body mass index by clinical reference values or median values. We developed Cox proportional hazard models incorporating the same risk adjusting variables to estimate the effect of short door to balloon time in patients with early presentation ( $\leq 2$ hours after symptom onset) and in those with delayed presentation ( $>2$ hours after symptom onset). We added an interaction variable between the time to presentation (early versus delayed) and door to balloon time (short versus long) in the model of the entire study population to assess whether the effect size of a door to balloon time of 90 minutes or less relative to more than 90 minutes differed between the two groups of patients with early and delayed presentations.

A physician (HS) and a statistician (TM) used JMP 7 and SAS 9.2 to do all the analyses. All the reported $\mathrm{P}$ values are two sided. We regarded a $\mathrm{P}$ value under 0.05 as statistically significant.

\section{Results}

\section{Presentation, baseline characteristics, and drugs according to onset to balloon time}

The median onset to balloon time in the entire study population was 4.2 (interquartile range 2.9-7.3) hours. The median times in the short and the delayed onset to balloon time groups were $2.4(2.0-2.7)$ hours and $5.5(4.0-9.4)$ hours. The rate of achievement of a door to balloon time of 90 minutes or less was significantly higher in the short onset to balloon time group than in the delayed onset to balloon time group (table $\Downarrow$ ).

Baseline characteristics differed significantly in several aspects between the short and delayed onset to balloon time groups (table $\Downarrow$ ). Patients with advanced age, previous stroke, and anaemia were more common in the delayed onset to balloon time group, whereas the short onset to balloon time group included more patients who were current smokers and had unstable haemodynamics such as cardiogenic shock (Killip class 4) at presentation. The delayed onset to balloon time group included more patients with multivessel disease and a culprit lesion of left circumflex artery, whereas a culprit lesion of left main coronary artery was more prevalent in the short onset to balloon time group (table $\downarrow$ ). The use of statins and $\beta$ blockers at discharge from hospital was significantly more prevalent in the short onset to balloon time group (table $\Downarrow$ ).

\section{Clinical outcomes: short versus delayed onset to balloon time}

The three year incidence of a composite of death and congestive heart failure in patients with short onset to balloon time was significantly lower than that in patients with delayed onset to balloon time (13.5\% (123 events/964 patients) v 19.2\%
(429/2427), $\mathrm{P}<0.001$; relative risk reduction 29.7\%) (fig 4ฟ, top left). An incremental increase in the three year incidence of a composite of death and congestive heart failure was apparent as the onset to balloon time increased from three hours or less to 12-24 hours (fig $4 \Downarrow$, top right). After adjustment for confounders, an onset to balloon time of three hours or less remained associated with a lower risk of a composite of death and congestive heart failure (adjusted hazard ratio 0.70, 95\% confidence interval 0.56 to $0.88 ; \mathrm{P}=0.002$ ). The three year incidence of all cause death in patients with short onset to balloon time was also significantly lower than that in patients with delayed onset to balloon time (10.4\% (94/964) v 14.6\% (323/2427), $\mathrm{P}=0.009$; relative risk reduction $28.8 \%$ ) (fig $4 \Downarrow$, bottom left) and tended to be lower than those in the remaining three categories with delayed onset to balloon time (fig $4 \Downarrow$, bottom right).

In the subgroup analysis according to the risk profile of the patients, the trend for better clinical outcome in patients with short onset to balloon time was consistent regardless of the presence or absence of risk factors such as heart failure, advanced age, anterior myocardial infarction, renal insufficiency, and multivessel disease, although the absolute differences in the incidences of events were greater in patients at high risk (fig $5 \Downarrow$ and fog $6 \Downarrow)$.

\section{Presentation, baseline characteristics, and drugs according to door to balloon time}

The median door to balloon time in the entire study population was 90 (60-132) minutes. The median time in the short and long door to balloon time groups were 60 (48-78) minutes and 132 (108-168) minutes. The onset to balloon time was significantly shorter in the short than in the long door to balloon time group-3.7 (2.5-6.5) hours versus 4.7 (3.3-7.8) hours $(\mathrm{P}<0.001)$. Patients with advanced age, body mass index below 25 , diabetes treated with insulin, heart failure, lower ejection fraction, severe mitral regurgitation, and previous myocardial infarction were more common in the long door to balloon time group, whereas the short door to balloon time group included more current smokers. The long door to balloon time group included more patients with multivessel disease and a culprit lesion of left circumflex artery, whereas a culprit lesion of left main coronary artery or proximal left descending artery was more prevalent in the short door to balloon time group. The use of statins and nitrates at hospital discharge was significantly more prevalent in the short door to balloon time group, whereas cilostazol and nicorandil were more often used in the long door to balloon time group (supplementary table B).

\section{Clinical outcomes: short versus long door to balloon time}

In the entire study population, we found no significant difference in the incidence of a composite of death and congestive heart failure $(16.7 \%(270 / 1671) v 18.4 \%(282 / 1720), \mathrm{P}=0.54$; relative risk reduction $9.2 \%)$ or all cause death $(12.4 \%(198 / 1671) v$ $14.4 \%$ (219/1720), $\mathrm{P}=0.21 ; 13.9 \%$ ) between the two groups of patients with short and long door to balloon time (fig $7 \Downarrow$, top). However, when we stratified patients by presentation time, short as compared with long door to balloon time was associated with significantly lower incidence of a composite of death and congestive heart failure in patients presenting early $(\leq 2$ hours after symptom onset) (11.9\% (74/883) v 18.1\% (147/655), $\mathrm{P}=0.01$; relative risk reduction $34.3 \%$ ), but not those presenting later (>2 hours after symptom onset) (19.7\% (196/788) v $18.7 \%$ (135/1065), $\mathrm{P}=0.44 ;-5.3 \%$ ) (fig $7 \Downarrow$, bottom). After adjusting 
for confounders, we found no significant difference in the risk of a composite of death and congestive heart failure between the two groups of patients with short and long door to balloon time (adjusted hazard ratio $0.98,0.78$ to $1.24 ; \mathrm{P}=0.87$ ). Short door to balloon time was independently associated with a lower risk of a composite of death and congestive heart failure in patients with early presentation (adjusted hazard ratio $0.58,0.38$ to $0.87 ; \mathrm{P}=0.009$ ) but not in those with delayed presentation $(1.57,1.12$ to $2.18 ; \mathrm{P}=0.008)$. A significant interaction existed between door to balloon time ( $\leq 90$ or $>90$ minutes) and the time to presentation ( $\leq 2$ or $>2$ hours after symptom onset) (interaction $\mathrm{P}=0.01$ ).

\section{Short term versus long term clinical outcomes}

Although the long term (three year) incidences of the principal outcome measure and all cause mortality were significantly lower in patients with short onset to balloon time, the short term (30 day) outcome did not differ between the short and delayed onset to balloon time groups in terms of all cause mortality $(5.0 \%(48 / 964) v 5.2 \%(125 / 2427), \mathrm{P}=0.82)$ and a composite of death and congestive heart failure $(5.2 \%$ (50/964) v 5.5\% (133/2427), $\mathrm{P}=0.71)$. A trend towards better clinical outcome with short door to balloon time in patients with early presentation was already apparent at 30 days both for all cause death $(4.3 \%(28 / 883) v 5.7 \%(50 / 655), \mathrm{P}=0.23)$ and for a composite of death and congestive heart failure $(4.3 \%$ (28/883) $v 5.9 \%(52 / 655), \mathrm{P}=0.16)$ (fig $8 \Downarrow)$.

\section{Discussion}

The main findings of this analysis are that a short onset to balloon time of less than three hours was associated with a lower risk of a composite of death and congestive heart failure up to three years of follow-up in patients with ST segment elevation myocardial infarction having primary percutaneous coronary intervention but that the benefit of a short door to balloon time of less than 90 minutes for clinical outcome was limited to patients who presented early.

\section{Comparison with other studies}

The large US national registry of myocardial infarction (NRMI) reported that short door to balloon time but not short onset to balloon time was associated with lower in-hospital mortality. ${ }^{67}$ However, consistent with the results from several smaller studies, our analysis showed a clear association between a short onset to balloon time of less than three hours and better long term clinical outcome. Theoretically, short onset to balloon time, meaning short total ischaemic time, would be expected to be associated with better clinical outcome, because experimental studies have suggested that the effect of reperfusion on myocardial salvage is markedly decreased within two or three hours after the onset of myocardial infarction. ${ }^{13}{ }^{15}$ In actual clinical practice, the correlation between short onset to balloon time and decreased mortality could be unclear because of the uncertainty about symptom onset, survivor bias, variations in the time course of development of myocardial necrosis, and very low in-hospital mortality in the era of primary percutaneous coronary intervention. One of the possible reasons for the discrepancy in the relation of onset to balloon time to clinical outcome between the NRMI study and our study might be a difference in the timeframe of endpoint evaluation - in-hospital event in the NRMI registry and three year event in this study. Clinical outcome at 30 days did not differ between the short and delayed onset to balloon time groups in our study. In-hospital mortality could be affected more by clinical conditions at presentation than by small differences in onset to balloon time or door to balloon time.

\section{Implications of study}

Guidelines emphasise the target of a door to balloon time of 90 minutes or less, and the rate at which this is achieved has improved in recent years. ${ }^{16}{ }^{17}$ The emphasis on the importance of short door to balloon time was mainly derived from the result of the NRMI, which suggested that short door to balloon time was associated with lower in-hospital mortality regardless of time from symptom onset to presentation. However, our study, in line with several previous studies, suggests that better in-hospital and long term outcome in patients with short door to balloon time was seen only in those patients who presented early..$^{11}$ The door to balloon time is a part of the onset to balloon time, and, theoretically, the relative importance of door to balloon time would decrease with increasing onset to balloon time. Flynn et al reported that an observed reduction in the door to balloon time between 2003 and 2008 did not lead to significant reduction in in-hospital mortality rates, suggesting that successful implementation of efforts to reduce door to balloon time has not resulted in the expected survival benefits. ${ }^{18}$

Therefore, we should focus more on reducing the onset to balloon time rather than focusing too much on the door to balloon time, although we should continue to make efforts to reduce the door to balloon time in patients presenting early. Further improvement in the outcome of patients with STEMI could be achieved by reducing the total ischaemic time with efforts such as social activities to raise awareness for patients to attend hospital early and improvement of pre-hospital care systems. Finally, all healthcare professionals, including primary care doctors, paramedics, acute physicians, and emergency department staff, as well as cardiologists, should make every effort to promote widespread awareness of the importance of shortening the onset to balloon time in saving the lives of patients with STEMI.

\section{Limitations of study}

This study has several limitations. Firstly, we could not exclude the influences of patients' recall bias for symptom onset. Therefore, we accept that exactly the same difficulty found in other studies regarding symptom onset applies to our own study and might partly account not only for the discordance of our findings compared with other studies but also for the internal discordance of late versus early benefit of short onset to balloon time. Secondly, clinical outcomes could have been affected by survivor bias and by variations in the time course of development of myocardial necrosis. Thirdly, risk adjustment by using dichotomised variables had the potential for residual confounding. In addition, treating some variables as continuous variables theoretically improves the power to detect differences. ${ }^{19}$ However, we consistently dichotomised the continuous variables in our reports based on the current registry because those dichotomised variables are more easily understood by general physicians. Finally, the huge differences in baseline characteristics between patients with early reperfusion and those with delayed reperfusion might limit the comparability of the groups, although we intended to adjust as extensively as possible to minimise the influence of unmeasured confounders.

\section{Conclusions}

Short onset to balloon time was associated with better three year clinical outcome in patients with STEMI having primary percutaneous coronary intervention, whereas the benefit of short 
door to balloon time was limited to patients with early presentation. Efforts to minimise onset to balloon time, including reduction in patient related delays, should be recommended to improve clinical outcome of STEMI patients.

We thank the members of the cardiac catheterisation laboratories of the participating centres and the clinical research coordinators. Contributors: TK, YN, YF, AN, SS, RT, KY, KN, TS, HM, MA, HT, TM, $\mathrm{HE}$, and SS developed the study design. HS, YN, TM, and TK did the data analysis and drafted the manuscript. TK obtained funding and supervised the study. All authors contributed to the interpretation of the data and revision of the manuscript. TK is the guarantor.

Funding: This study was supported by the Pharmaceuticals and Medical Devices Agency (PMDA) in Japan. The PMDA had no role in the study design; in the collection, analysis, and interpretation of data; in the writing of the reports; or in the decision to submit the article for publication.

Competing interests: All authors have completed the Unified Competing Interest form at www.icmje.org/coi_disclosure.pdf (available on request from the corresponding author) and declare: no support from any organisation for the submitted work; no financial relationships with any organisations that might have an interest in the submitted work in the previous three years; no other relationships or activities that could appear to have influenced the submitted work.

Ethical approval: The protocol for the study was approved by the human research ethics committees of the Kyoto University Graduate School of Medicine.

Data sharing: No additional data available.

1 Gibson C, Pride Y, Frederick P, Pollackjr C, Canto J, Tiefenbrunn A, et al. Trends in reperfusion strategies, door-to-needle and door-to-balloon times, and in-hospital mortality among patients with ST-segment elevation myocardial infarction enrolled in the national registry of myocardial infarction from 1990 to 2006. Am Heart J 2008;156:1035-44.

2 Eagle KA, Nallamothu BK, Mehta RH, Granger CB, Steg PG, Van de Werf F, et al. Trends in acute reperfusion therapy for ST-segment elevation myocardial infarction from 1999 to 2006: we are getting better but we have got a long way to go. Eur Heart $J$ 2008;29:609-17.

3 Antman EM, Anbe DT, Armstrong PW, Bates ER, Green LA, Hand M, et al. ACC/AHA guidelines for the management of patients with ST-elevation myocardial infarction: a report of the American College of Cardiology/American Heart Association Task Force on Practice Guidelines (Committee to Revise the 1999 Guidelines for the Management of Patients with Acute Myocardial Infarction). Circulation 2004;110:e82-292.

4 Antman EM, Hand M, Armstrong PW, Bates ER, Green LA, Halasyamani LK, et al. 2007 focused update of the ACC/AHA 2004 guidelines for the management of patients with ST-elevation myocardial infarction: a report of the American College of Cardiology/American Heart Association Task Force on Practice Guidelines: developed in collaboration with the Canadian Cardiovascular Society endorsed by the American Academy of Family Physicians. Circulation 2007:117:296-329.

5 Van de Werf F, Bax J, Betriu A, Blomstrom-Lundqvist C, Crea F, Falk V, et al. Management of acute myocardial infarction in patients presenting with persistent ST-segment elevation: the task force on the management of ST-segment elevation acute myocardial infarction of the European Society of Cardiology. Eur Heart J 2008;29:2909-45.

6 Cannon CP. Relationship of symptom-onset-to-balloon time and door-to-balloon time with mortality in patients undergoing angioplasty for acute myocardial infarction. JAMA 2000:283:2941-7.

7 McNamara R, Wang Y, Herrin J, Curtis J, Bradley E, Magid D, et al. Effect of door-to-balloon time on mortality in patients with ST-segment elevation myocardial infarction. J Am Coll Cardiol 2006;47:2180-6.

8 De Luca G, Suryapranata H, Zijlstra F, van't Hof AWJ, Hoorntje JCA, Gosselink ATM, et al. Symptom-onset-to-balloon time and mortality in patients with acute myocardial infarction treated by primary angioplasty. J Am Coll Cardiol 2003;42:991-7.

9 Brodie BR, Stuckey TD, Wall TC, Kissling G, Hansen CJ, Muncy DB, et al. Importance of time to reperfusion for 30 -day and late survival and recovery of left ventricular function after primary angioplasty for acute myocardial infarction. J Am Coll Cardiol 1998;32:1312-9.

10 Brodie B, Hansen C, Stuckey T, Richter S, Versteeg D, Gupta N, et al. Door-to-balloon time with primary percutaneous coronary intervention for acute myocardial infarction impacts late cardiac mortality in high-risk patients and patients presenting early after the onset of symptoms. J Am Coll Cardiol 2006;47:289-95.

11 Brodie BR, Gersh BJ, Stuckey T, Witzenbichler B, Guagliumi G, Peruga JZ, et al. When is door-to-balloon time critical? Analysis from the HORIZONS-AMI (Harmonizing Outcomes with Revascularization and Stents in Acute Myocardial Infarction) and CADILLAC (Controlled Abciximab and Device Investigation to Lower Late Angioplasty Complications) tials. J Am Coll Cardiol 2010;56:407-13.

12 Hannan EL, Zhong Y, Jacobs AK, Holmes DR, Walford G, Venditti FJ, et al. Effect of onset-to-door time and door-to-balloon time on mortality in patients undergoing percutaneous coronary interventions for ST-segment elevation myocardial infarction. Am J Cardiol 2010;106:143-7.

13 Gersh BJ. Pharmacological facilitation of primary percutaneous coronary intervention for acute myocardial infarction: is the slope of the curve the shape of the future? JAMA 2005;293:979-86.

14 Boden W, Eagle K, Granger C. Reperfusion strategies in acute ST-segment elevation myocardial infarction: a comprehensive review of contemporary management options. $J$ Am Coll Cardiol 2007;50:917-29.

15 Gersh BJ, Anderson JL. Thrombolysis and myocardial salvage: results of clinical trials and the animal paradigm — paradoxic or predictable? Circulation 1993;88:296-306.

$16 \mathrm{Krumholz} \mathrm{H.} \mathrm{A} \mathrm{campaign} \mathrm{to} \mathrm{improve} \mathrm{the} \mathrm{timeliness} \mathrm{of} \mathrm{primary} \mathrm{percutaneous} \mathrm{coronary}$ intervention door-to-balloon: an alliance for quality. JACC Cardiovasc Interv 2008;1:97-104

17 Bradley EH, Nallamothu BK, Herrin J, Ting HH, Stern AF, Nembhard IM, et al. National efforts to improve door-to-balloon time results from the door-to-balloon alliance. J Am Coll Cardiol 2009;54:2423-9.

18 Flynn A, Moscucci M, Share D, Smith D, LaLonde T, Changezi H, et al. Trends in door-to-balloon time and mortality in patients with ST-elevation myocardial infarction undergoing primary percutaneous coronary intervention. Arch Intern Med 2010;170:1842-9.

19 Altman DG, Royston P. The cost of dichotomising continuous variables. BMJ 2006;332:1080.

Accepted: 05 April 2012

\section{Cite this as: BMJ 2012;344:e3257}

This is an open-access article distributed under the terms of the Creative Commons Attribution Non-commercial License, which permits use, distribution, and reproduction in any medium, provided the original work is properly cited, the use is non commercial and is otherwise in compliance with the license. See: http://creativecommons.org/licenses/bync/2.0/ and http://creativecommons.org/licenses/by-nc/2.0/legalcode. 


\section{What is already known on this topic}

Results from previous studies are quite inconsistent regarding the relation of symptom onset to balloon time and clinical outcomes in patients with ST segment elevation myocardial infarction

The time to evaluate endpoints varied widely between these different studies

Little is known about the relation of onset to balloon time with long term clinical outcomes in actual clinical practice

\section{What this study adds}

A clear association has been shown between a short onset to balloon time of less than three hours and better long term (three year) clinical outcomes

The benefit of short door to balloon time was limited to patients who presented early

Further improvement in the outcome of patients with ST segment elevation myocardial infarction could be achieved by reducing the total ischaemic time with various efforts

\section{Table}

\begin{tabular}{|c|c|c|c|c|}
\hline & Entire cohort ( $n=3391)$ & $\begin{array}{c}\text { Onset to balloon time } \leq 3 \\
\text { hours }(n=964)\end{array}$ & $\begin{array}{l}\text { Onset to balloon time }>3 \text { hours } \\
\qquad(n=2427)\end{array}$ & $P$ value \\
\hline \multicolumn{5}{|l|}{ Baseline characteristics } \\
\hline Mean (SD) age (years) & $67.5(12.2)$ & $65.3(12.0)$ & $68.3(12.2)$ & $<0.001$ \\
\hline Male sex & $2512(74)$ & $768(80)$ & $1744(72)$ & $<0.001$ \\
\hline Mean (SD) body mass index $\left(\mathrm{kg} / \mathrm{m}^{2}\right)$ & $23.5(3.4)$ & $23.8(3.4)$ & $23.5(3.4)$ & 0.01 \\
\hline Hypertension & $2660(78)$ & $741(77)$ & $1919(79)$ & 0.16 \\
\hline Diabetes mellitus & $1066(31)$ & $311(32)$ & $755(31)$ & 0.51 \\
\hline Treated with insulin & $145(4.3)$ & $41(4.3)$ & $104(4.3)$ & 0.97 \\
\hline Current smoker & $1380(41)$ & $445(46)$ & $935(39)$ & $<0.001$ \\
\hline Heart failure & $1050(31)$ & $304(32)$ & $746(31)$ & 0.65 \\
\hline Mean (SD) ejection fraction & $52.6(12.6)$ & $53.6(12.7)$ & $52.1(12.6)$ & 0.005 \\
\hline Previous myocardial infarction & $293(8.6)$ & $101(10)$ & $192(7.9)$ & 0.02 \\
\hline Previous stroke (with symptoms) & $288(8.5)$ & $64(6.6)$ & $224(9.2)$ & 0.01 \\
\hline Peripheral vascular disease & $102(3.0)$ & $25(2.6)$ & $77(3.2)$ & 0.37 \\
\hline Median (IQR) eGFR $\left(\mathrm{mL} / \mathrm{min} / 1.73 \mathrm{~m}^{2}\right)^{\star}$ & $69.4(54.2-85.4)$ & $68.0(53.4-82.0)$ & $70.3(54.5-86.5)$ & 0.03 \\
\hline Atrial fibrillation & $314(9.3)$ & $98(10)$ & $216(8.9)$ & 0.26 \\
\hline Anaemia (haemoglobin $<11.0 \mathrm{~g} / \mathrm{dL}$ ) & $309(9.1)$ & $63(6.5)$ & $246(10)$ & $<0.001$ \\
\hline Chronic obstructive pulmonary disease & $106(3.1)$ & $27(2.8)$ & $79(3.3)$ & 0.49 \\
\hline \multicolumn{5}{|l|}{ Presentation } \\
\hline Median (IQR) hours from onset to presentation & $2.4(1.1-5.1)$ & $0.9(0.6-1.3)$ & $3.4(2.0-7.0)$ & $<0.001$ \\
\hline Median (IQR) hours from onset to balloon & $4.2(2.9-7.3)$ & $2.4(2.0-2.7)$ & $5.5(4.0-9.4)$ & $<0.001$ \\
\hline \multicolumn{5}{|l|}{ Hours from onset to balloon: } \\
\hline$<3$ & $964(28)$ & $964(100)$ & $0(0)$ & \multirow[t]{4}{*}{$<0.001$} \\
\hline $3-6$ & $1359(40)$ & $0(0)$ & $1359(56)$ & \\
\hline $6-12$ & $659(19)$ & $0(0)$ & $659(27)$ & \\
\hline $12-24$ & $409(12)$ & $0(0)$ & $409(17)$ & \\
\hline Median (IQR) minutes from door to balloon & $90(60-132)$ & $78(54-102)$ & $102(66-150)$ & $<0.001$ \\
\hline$\leq 90$ minutes from door to balloon & $1720(51)$ & $635(66)$ & $1085(45)$ & $<0.001$ \\
\hline \multicolumn{5}{|l|}{ Haemodynamics: } \\
\hline Killip class 1 & $2551(75)$ & 700 (73) & $1851(76)$ & \multirow[t]{4}{*}{$<0.001$} \\
\hline Killip class 2 & $283(8.3)$ & $65(6.7)$ & $218(9.0)$ & \\
\hline Killip class 3 & $85(2.5)$ & $17(1.8)$ & $68(2.8)$ & \\
\hline Killip class 4 & $472(14)$ & $182(19)$ & $290(12)$ & \\
\hline Intra-aortic balloon pump use & $528(16)$ & $172(18)$ & $356(15)$ & 0.02 \\
\hline
\end{tabular}


Table 1 (continued)

\begin{tabular}{|c|c|c|c|c|}
\hline & Entire cohort $(n=3391)$ & $\begin{array}{c}\text { Onset to balloon time } \leq 3 \\
\text { hours }(n=964)\end{array}$ & $\begin{array}{l}\text { Onset to balloon time }>3 \text { hours } \\
\qquad(n=2427)\end{array}$ & $P$ value \\
\hline \multicolumn{5}{|l|}{ Angiographic characteristics } \\
\hline \multicolumn{5}{|l|}{ Infarct related artery location: } \\
\hline Left anterior descending artery & $1577(47)$ & $432(45)$ & $1145(47)$ & \multirow[t]{5}{*}{$<0.001$} \\
\hline Left circumflex artery & $331(9.8)$ & $72(7.5)$ & $259(11)$ & \\
\hline Right coronary artery & $1395(41)$ & $424(44)$ & $971(40)$ & \\
\hline Left main coronary artery & $73(2.2)$ & $32(3.3)$ & $41(1.7)$ & \\
\hline Coronary artery bypass graft & $15(0.4)$ & $4(0.4)$ & $11(0.5)$ & \\
\hline Multivessel disease & $1714(51)$ & $455(47)$ & $1259(52)$ & 0.01 \\
\hline \multicolumn{5}{|l|}{ Drugs at discharge } \\
\hline Thienopyridine & $3257(96)$ & $926(96)$ & $2331(96)$ & 0.99 \\
\hline Aspirin & $3348(99)$ & $950(99)$ & $2398(99)$ & 0.55 \\
\hline Statins & $1823(54)$ & $549(57)$ & $1274(52)$ & 0.02 \\
\hline$\beta$ blockers & $1381(41)$ & $419(43)$ & $962(40)$ & 0.04 \\
\hline ACE inhibitor/ARB & $2447(72)$ & $705(73)$ & $1742(72)$ & 0.43 \\
\hline Nitrates & $1020(30)$ & $294(31)$ & $726(30)$ & 0.74 \\
\hline Calcium channel blockers & $649(19)$ & $189(20)$ & $460(19)$ & 0.66 \\
\hline
\end{tabular}

$\mathrm{ACE}=$ angiotensin converting enzyme; $\mathrm{ARB}=$ angiotensin receptor blocker; $\mathrm{IQR}=$ interquartile range.

*Estimated glomerular filtration rate calculated by modification of diet in renal disease formula modified for Japanese patients. 


\section{Figures}

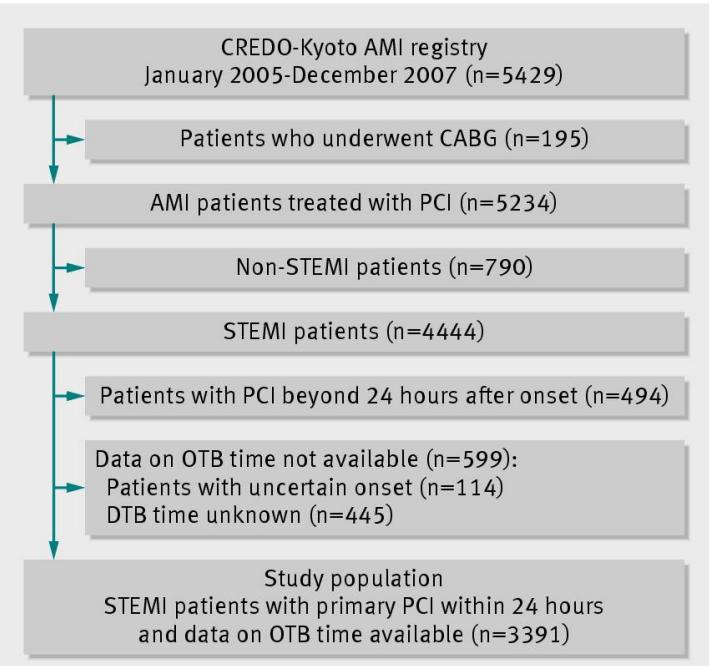

Fig 1 Flow chart for selection of study population. AMI=acute myocardial infarction; DTB=door to balloon time; OTB=onset to balloon; $\mathrm{PCl}=$ percutaneous coronary intervention; STEMI=ST segment elevation myocardial infarction

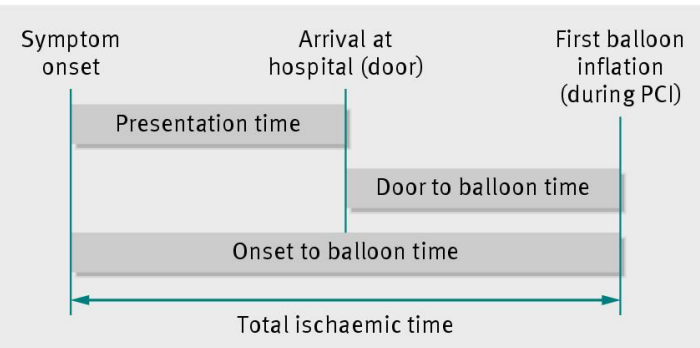

Fig 2 Schema for timeframe from symptom onset to primary percutaneous coronary intervention (PCl) 

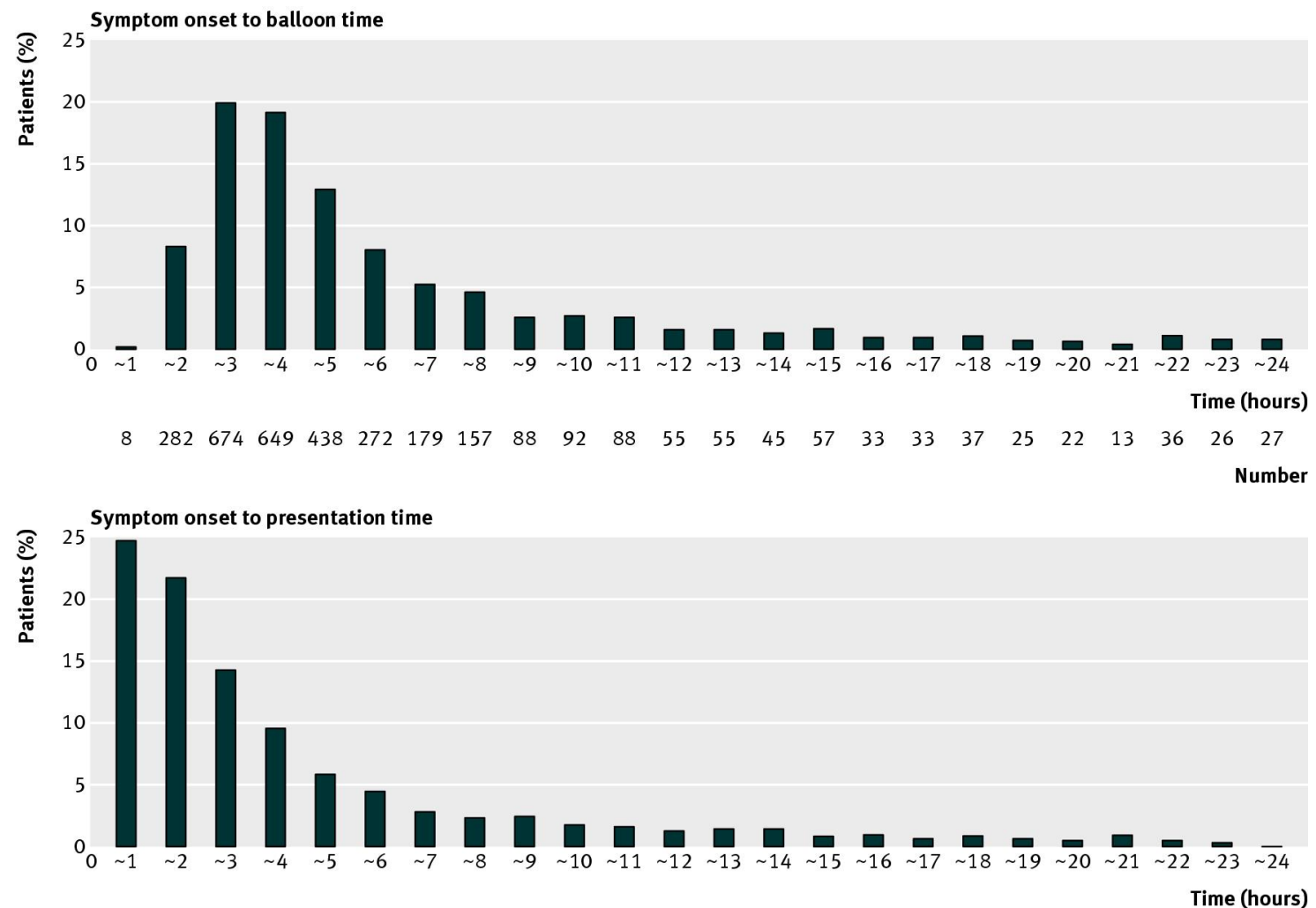

$\begin{array}{lllllllllllllllllllllllll}803 & 735 & 482 & 322 & 197 & 154 & 95 & 78 & 81 & 58 & 54 & 40 & 48 & 46 & 28 & 32 & 22 & 28 & 19 & 14 & 31 & 14 & 10 & 0\end{array}$

Number

Fig 3 Distribution of symptom onset to balloon time (left); distribution of symptom onset to hospital presentation time (right) 

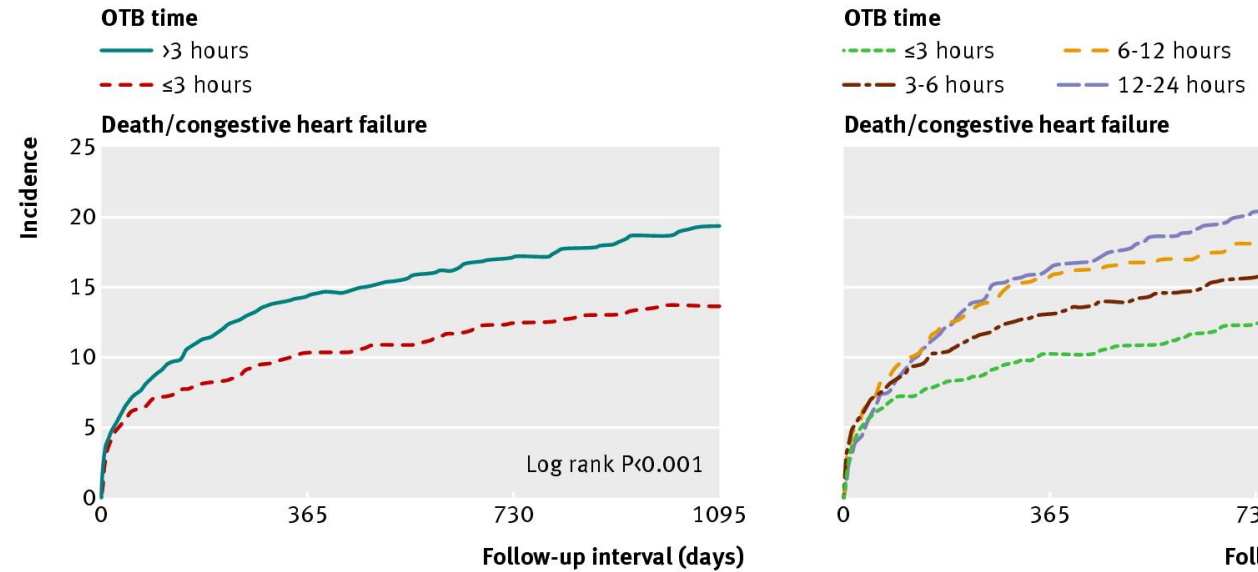

$--3-6$ hours $\quad-12-24$ hours

Death/congestive heart failure

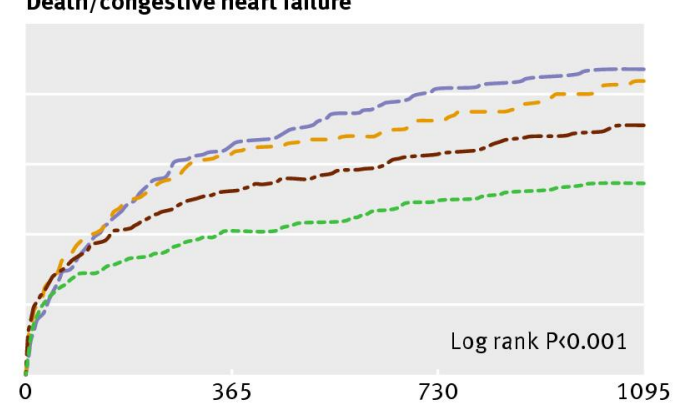

Follow-up interval (days)

Interval 0 day 30 days 1 year 2 years 3 years No of patients at risk $\leq 3$ hours

$\begin{array}{lrrrrr} & 964 & 907 & 835 & 653 & 349 \\ \text { >3 hours } & & & & & \\ & 2427 & 2262 & 1973 & 1528 & 839\end{array}$
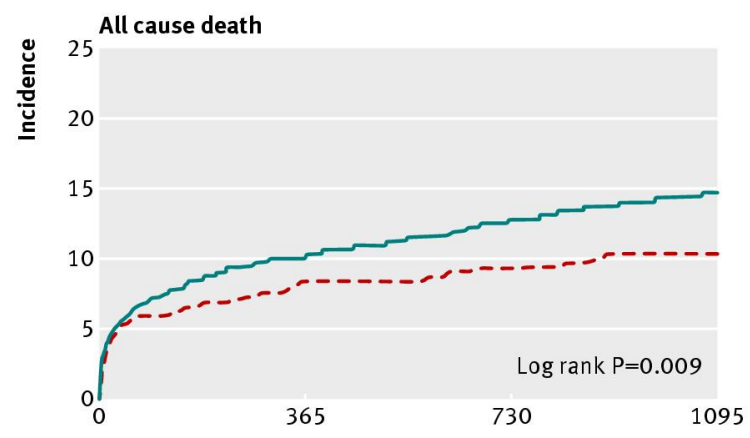

Follow-up interval (days)

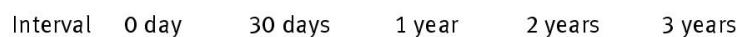

No of patients at risk $\leq 3$ hours

$\begin{array}{lrrrrr} & 964 & 909 & 853 & 679 & 365 \\ \text { 33 hours } & 2427 & 2270 & 2067 & 1611 & 891\end{array}$

No of patients at risk $\leq 3$ hours

No of patients at risk

$\leq 3$ hours

$\begin{array}{llllll} & 964 & 907 & 835 & 653 & 349\end{array}$

$\begin{array}{lllll}1359 & 1265 & 1127 & 890 & 490\end{array}$

$\begin{array}{rrrrr}659 & 614 & 530 & 401 & 224 \\ 12-24 \text { hours } & & & & \\ 409 & 383 & 316 & 237 & 125\end{array}$

All cause death

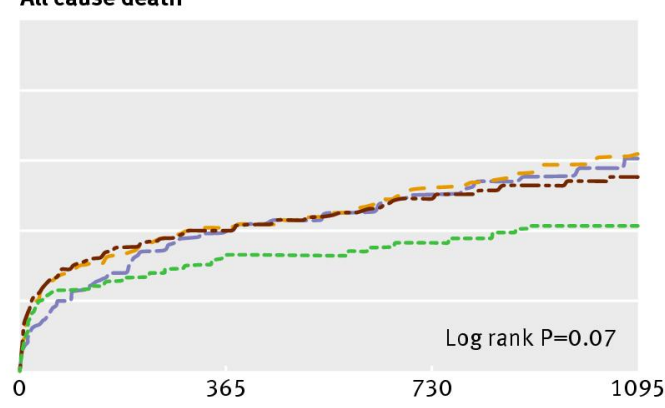

Follow-up interval (days)

$\begin{array}{rcccc}964 & 909 & 853 & 679 & 365 \\ \begin{array}{r}\text { 3-6 hours } 1359 \\ \text { 6-12 hours } 659\end{array} & 1270 & 1166 & 924 & 515 \\ \begin{array}{r}12-24 \text { hours } \\ 409\end{array} & 386 & 363 & 430 & 240 \\ & & 333 & 257 & 136\end{array}$

Fig 4 Clinical outcomes according to symptom onset to balloon time. Cumulative incidence of composite of death and congestive heart failure (CHF) was compared between two groups of patients with onset to balloon (OTB) time within and over three hours (top left) and among four OTB time categories (top right). Cumulative incidences of all cause death were also compared between two groups of patients with OTB time within and over three hours (bottom left) and among four OTB time categories (bottom right) 


\section{OTB time}

$\longrightarrow 3$ hours

$$
---\leq 3 \text { hours }
$$$$
\text { 몸 }
$$

\section{Patients with congestive heart failure}

ํㅡㄹ
$>3$ hours $(n=576)$
$\leq 3$ hours $(n=264)$
Log rank $\mathrm{P}=0.007$

60

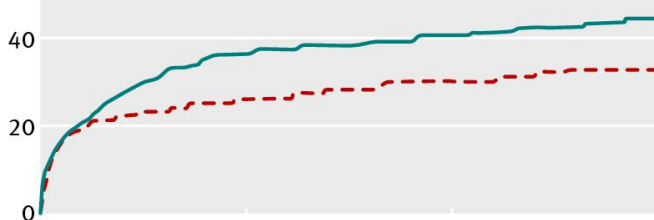

몷

\section{Patients $\geq 75$ years old}

$$
\begin{aligned}
& \quad 30 \text { hours }(n=813) \\
& \leq 3 \text { hours }(n=226)
\end{aligned}
$$

60

40

20
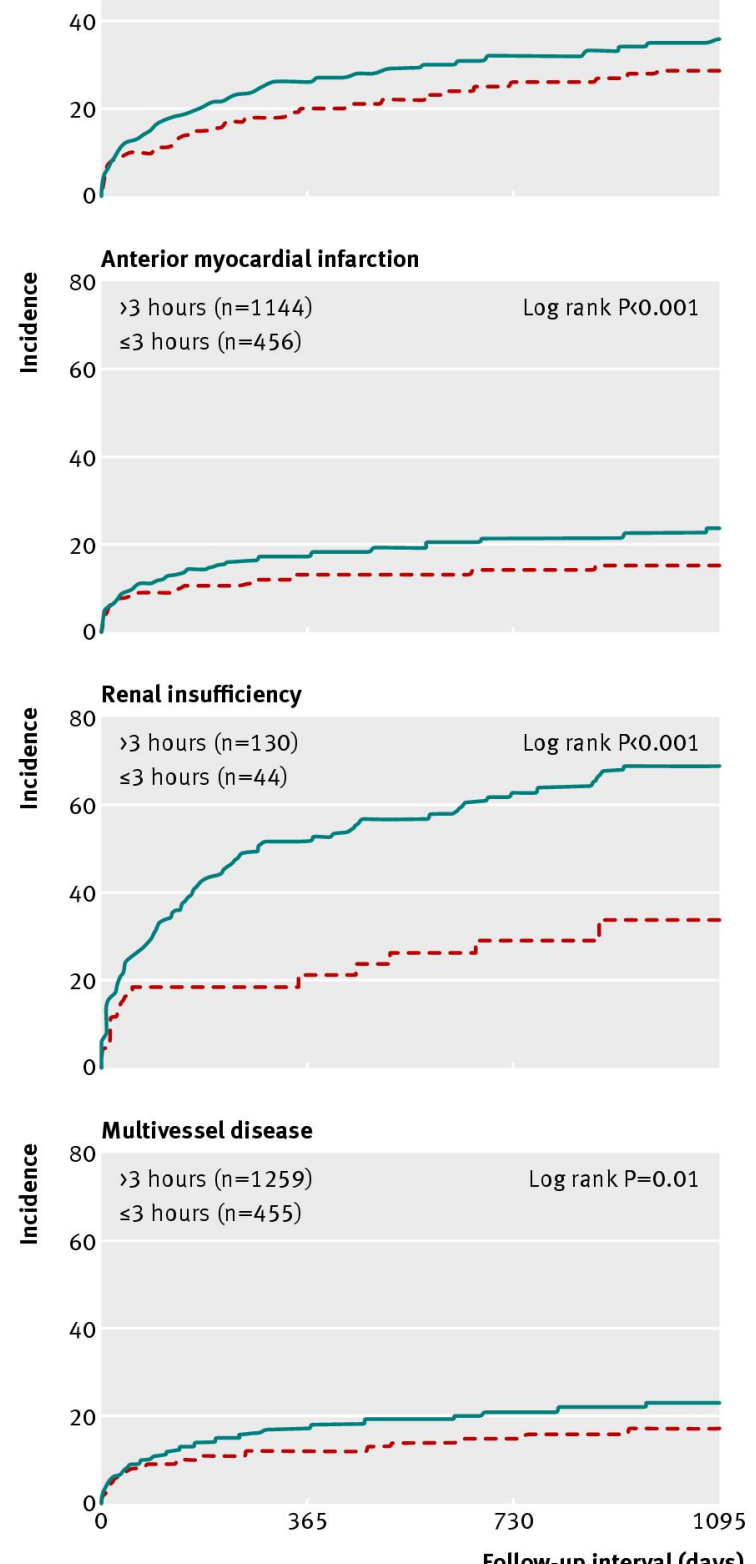

\section{Patients without congestive heart failure}

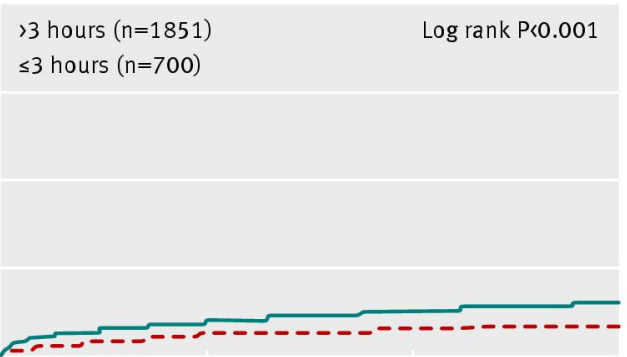

Patients $\$ 75$ years old

$>3$ hours $(n=1614)$

$\leq 3$ hours $(n=738)$

Log rank $\mathrm{P}=0.17$

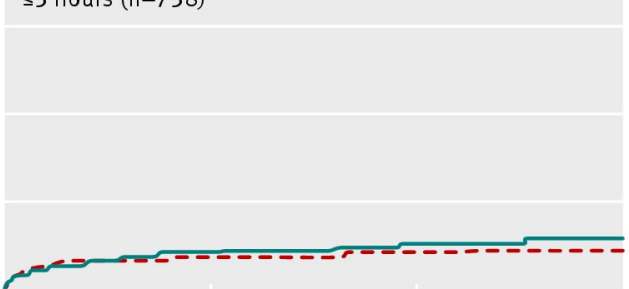

\section{Non-anterior myocardial infarction}

$>3$ hours $(\mathrm{n}=1283$ )

$\leq 3$ hours $(n=508)$

Log rank $\mathrm{P}=0.17$

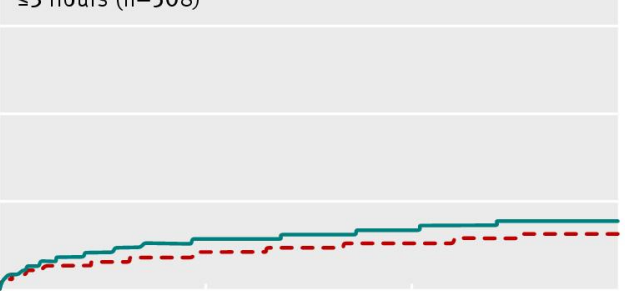

Non-renal insufficiency

$>3$ hours $(n=2297)$

$\leq 3$ hours $(n=920)$

Log rank $P=0.02$

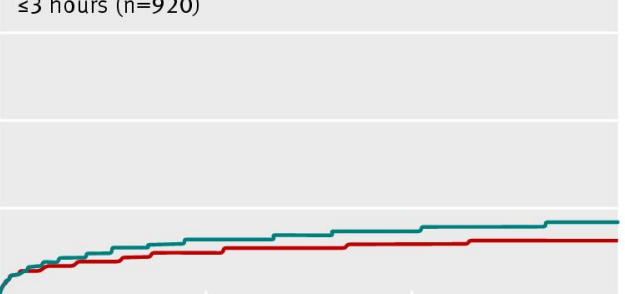

\section{Single vessel disease}

$>3$ hours $(\mathrm{n}=1168)$

$\leq 3$ hours $(n=509)$

$\log \operatorname{rank} P=0.04$

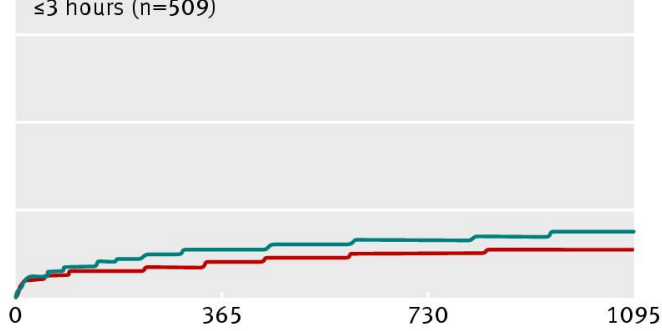

Follow-up interval (days)

Fig 5 Cumulative incidences of composite of death and congestive heart failure according to symptom onset to balloon (OTB) time stratified by risk profile of patients 


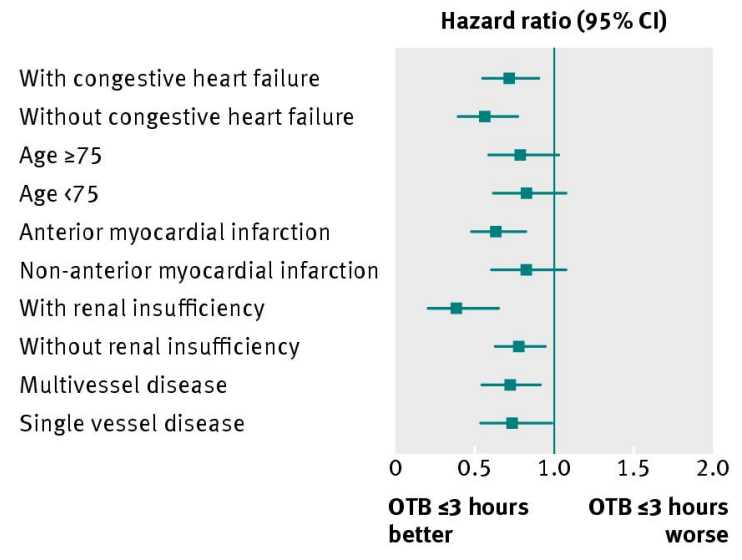

Fig 6 Hazard ratios of short versus delayed symptom onset to balloon (OTB) time for composite of death and congestive heart failure stratified by risk profile of patients 
DTB time

$\longrightarrow>90$ minutes

- - $\leq 90$ minutes

\section{Entire cohort}

Death/congestive heart failure

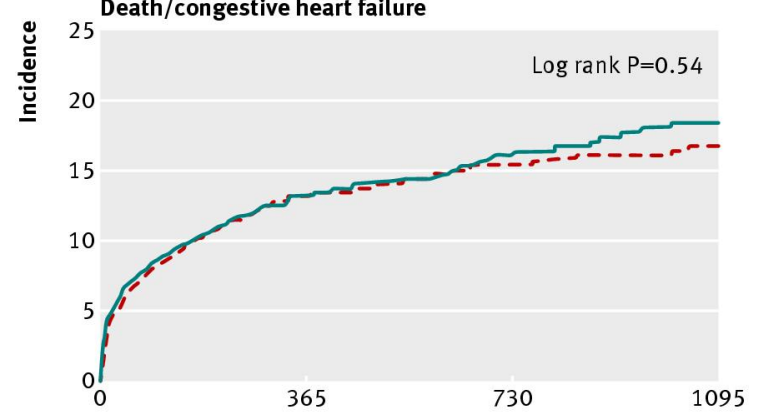

Follow-up interval (days)
Entire cohort

All cause death

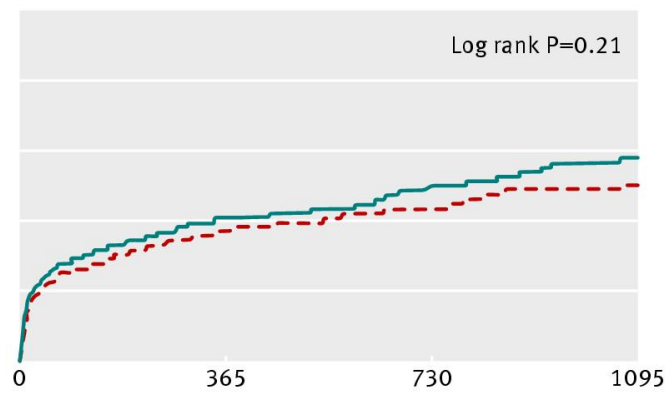

Follow-up interval (days)

Interval 0 day 30 days 1 year 2 years 3 years No of patients at risk

$\geq 90$ minutes

$\begin{array}{lllll}1671 & 1557 & 1378 & 1073 & 603\end{array}$

$\leq 90$ minutes

$\begin{array}{lllll}1720 & 1612 & 1430 & 1108 & 585\end{array}$

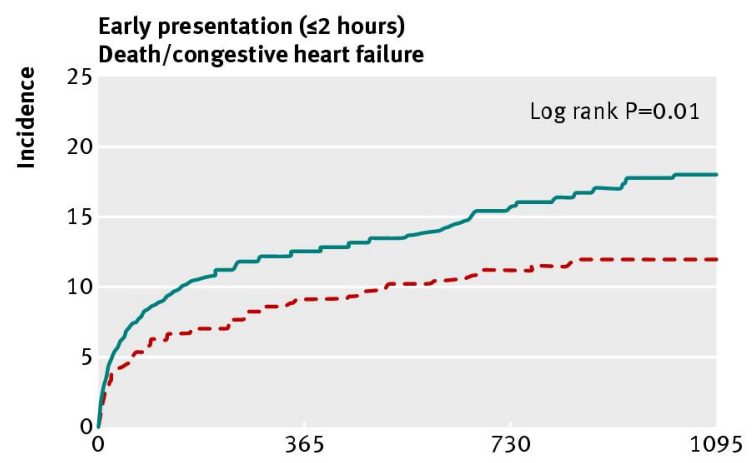

Follow-up interval (days)

Interval 0 day 30 days 1 year 2 years 3 years

No of patients at risk

$\geq 90$ minutes

$\begin{array}{lllll}883 & 824 & 740 & 590 & 316 \\ \leq 90 \text { minutes } & & & & \end{array}$

$\quad 0$ day
No of patients at risk
$\geq 90$ minutes

Fig 7 Clinical outcomes according to door to balloon time. Cumulative incidences of composite of death and congestive heart failure (CHF) (top left) and all cause death (top right) were compared between two groups of patients with door to balloon (DTB) time within and over 90 minutes in entire cohort. Cumulative incidences of composite of death and CHF were also compared between two groups of patients with DTB time within and over 90 minutes in patients with early presentation ( $\leq 2$ hours after symptom onset) (bottom left) and in those with delayed presentation ( $>2$ hours after symptom onset) (bottom right) 
OTB time and clinical outcomes

$\square$ OTB $\leq 3$ hours

$\square$ OTB $>3$ hours
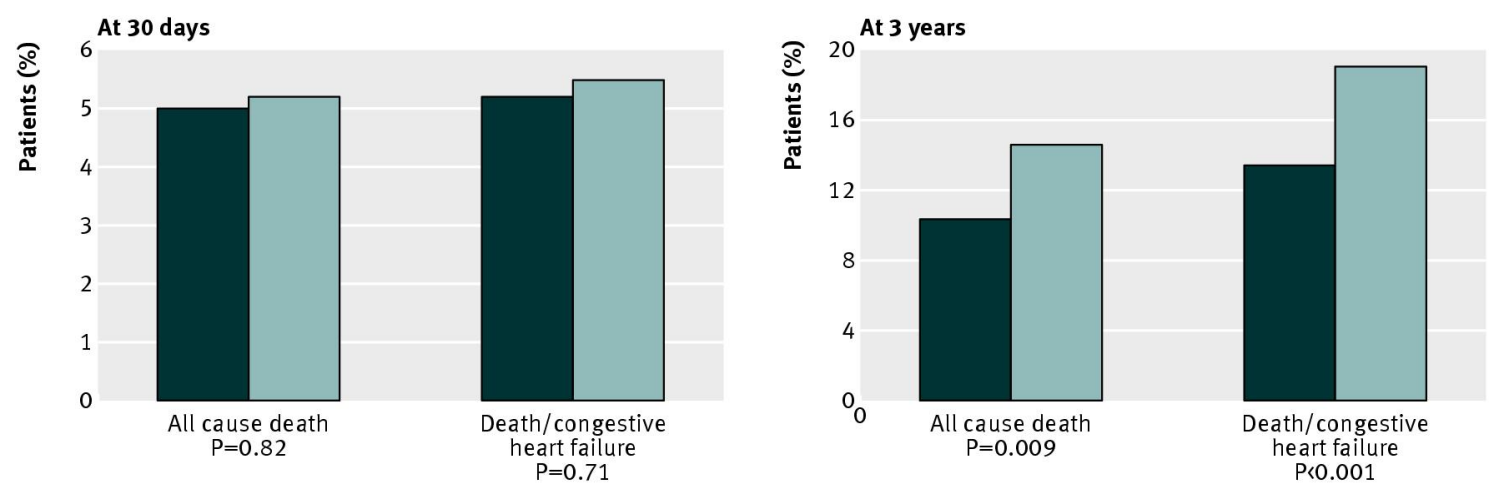

DTB time and clinical outcomes

\section{$\square$ DTB $\leq 90$ minutes}

$\square$ DTB $>90$ minutes
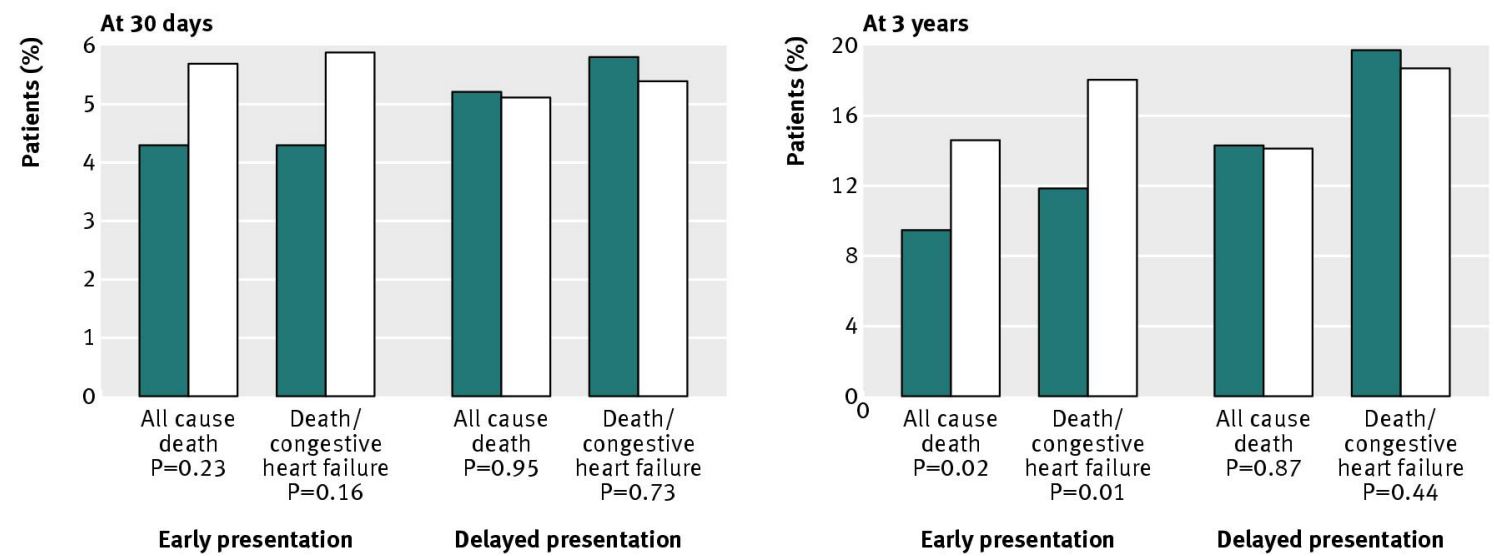

Fig 8 Clinical outcomes at 30 days and at 3 years according to symptom onset to balloon time (left), and according to door to balloon time (right) 